\title{
Calculation of Transient Excitation of Ship Hulls by Finite Difference Methods
}

\author{
By Harry Polachek
}

1. Introduction. A system of finite difference equations based on the non-uniform beam theory is developed for use in the calculation of the response of a ship hull to transient forces. The conditions for stability of these equations (and hence the conditions for validity of the numerical results) are derived. The feasibility of the method is tested by the solution of a vibration problem for a specific hull, the details of which are discussed in [1] and [2]. The use of this method lends itself to the solution of a wide class of problems related to the structural design of vesse] hulls or other structures subject to transient forces. The solution has been programmed and carried out on the Bureau of Ships UNIVAC System, Applied Mathematics Laboratory, David Taylor Model Basin.

2. Governing Equations. The equations governing the motion of a ship hull based on uniform and non-uniform beam theory as developed by Timoshenko [3] and others are discussed in considerable detail by R. T. McGoldrick and V. L. Russo in [4]. A finite difference method for obtaining numerical solutions to these equations is presented here. For this purpose, the equations describing the damped vertical (or torsion-free horizontal) excitation of a ship hull subjected to a transient force will be used. The results may be directly extended to more general types of motion. The system of partial differential equations describing this type of motion, as given in [4] is:

$$
\begin{gathered}
\mu \frac{\partial^{2} y}{\partial t^{2}}+c \frac{\partial y}{\partial t}+\frac{\partial V}{\partial x}=P(x, t) \\
I_{\mu z} \frac{\partial^{2} \gamma}{\partial t^{2}}+V-\frac{\partial M}{\partial x}=0 \\
M=(E I) \frac{\partial \gamma}{\partial x} \\
V=(K A G) \gamma-(K A G) \frac{\partial y}{\partial x}
\end{gathered}
$$

where,

$t \quad=$ time

$x \quad=$ distance coordinate along the longitudinal axis of a vessel

$y=$ displacement normal to the longitudinal axis

$\gamma \quad=$ rotation of transverse section about an axis normal to the $(x y)$ plane ( $z$-axis)

$M \quad=$ bending moment

$V \quad=$ net shear force in $y$ direction

Received March 2, 1959. 


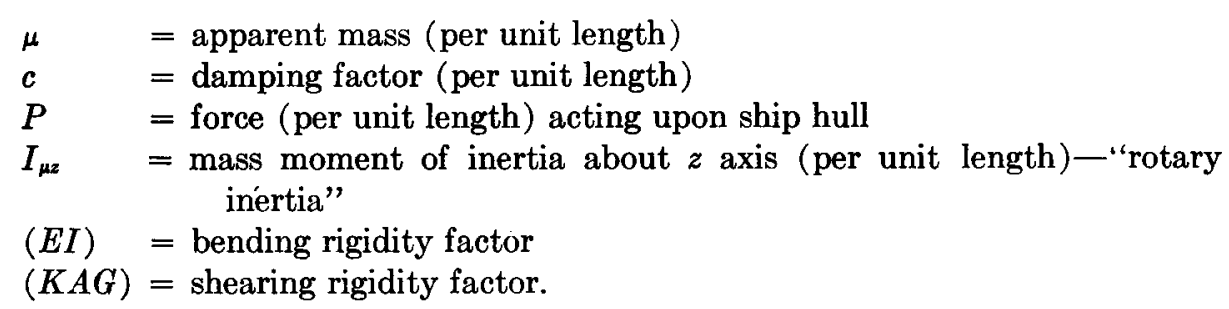

3. Finite Difference Representation. The set of equations (1) to (4) constitutes a system of partial differential equations which is assumed to govern the motion of the hull of a vessel, as simulated by a freely vibrating non-uniform beam. If the state of motion of the ship hull is known at any time $t_{0}$, it is possible by obtaining the solution to these equations to determine its motion at any subsequent time. This system of equations may be represented approximately in finite difference form by replacing the partial derivatives by equivalent ratios of small finite increments. In making this substitution we will use the following notation:

$t_{0}$ - initial time

$x_{0}$ - initial position

$\Delta t=$ increment in time

$\Delta x=$ increment in length

$\Delta y, \Delta \gamma, \Delta M, \Delta \cdot V=$ corresponding increments in the dependent variables $y, \gamma$, $M$ and $V$, respectively

$x_{n}=x_{0}+n \Delta x$

$y_{n+\frac{1}{2}}^{m}=y\left[x_{0}+\left(n+\frac{1}{2}\right) \Delta x, t_{0}+m \Delta t\right]=$ value of $y$ at the position

$x_{0}+\left(n+\frac{1}{2}\right) \Delta x$ and at the time $t_{0}+m \Delta t,\left\{\begin{array}{l}n=0,1,2 \cdots \\ m=0,1,2 \cdots\end{array}\right.$

$M_{n}^{m+1}=M\left[x_{0}+n \Delta x, t_{0}+(m+1) \Delta t\right]=$ value of $M$ at the position

$x_{0}+n \Delta x$ and at the time $t_{0}+(m+1) \Delta t ;\left\{\begin{array}{l}n=0,1,2 \cdots \\ m=0,1,2 \cdots\end{array}\right.$

Similar notation will be used for other variables and factors in the equations. For quantities which do not vary with time, the superscript will be omitted.

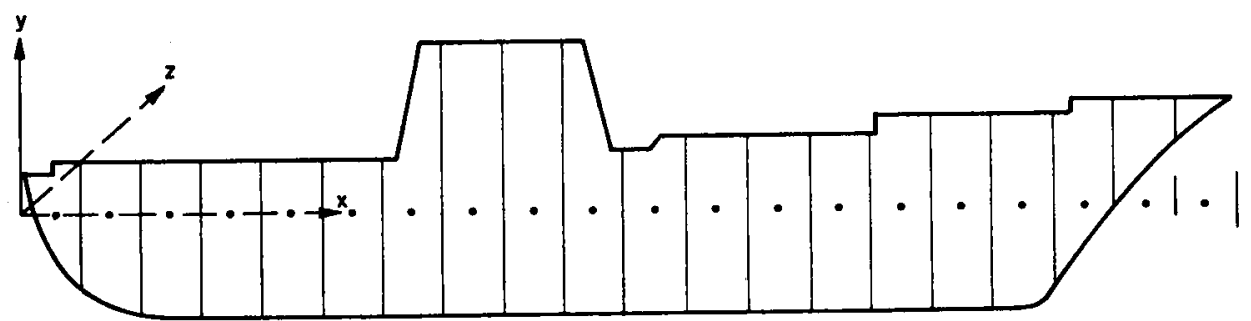

Frg. 1a.-Ship hull.

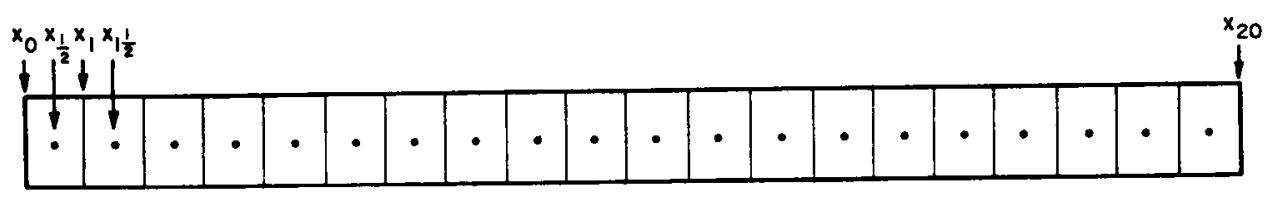

Fra. 1b.-Representation by non-uniform segmented beam. 
Figures (1a) and (1b) show the representation of a ship hull by means of a non-uniform beam and its division in twenty increments, as used in the initial solution. Using the above notation, the displacement of the hull at the position $x_{2}$ (the boundary point between the second and third increments) at the end of the $m$ th time increment, for instance, will be represented by ${y_{2}}^{m}$; its displacement at the center of the second increment will be represented by $y_{1 \frac{1}{2}}^{m}$. The average mass per unit length at the center of the twentieth increment will similarly be represented by $\mu_{19.5}$.

To solve the above system of equations we propose to make the following specific finite difference substitutions in the system of equations (1) to (4):

$$
\begin{aligned}
& \left(\frac{\partial^{2} y}{\partial t^{2}}\right)_{n+1}^{m}=\frac{y_{n+\frac{1}{2}}^{m+1}-2 y_{n+\frac{1}{3}}^{m}+y_{n+\frac{1}{3}}^{m-1}}{(\Delta t)^{2}} \\
& \left(\frac{\partial^{2} \gamma}{\partial t^{2}}\right)_{n+1}^{m}=\frac{\gamma_{n+\frac{1}{3}}^{m+1}-2 \gamma_{n+1}^{m}+\gamma_{n+\frac{1}{3}}^{m-1}}{(\Delta t)^{2}} \\
& \left(\frac{\partial y}{\partial t}\right)_{n+1}^{m}=\frac{y_{n+\frac{1}{3}}^{m}-y_{n+\frac{1}{2}}^{m-1}}{(\Delta t)}
\end{aligned}
$$

$$
\left(\frac{\partial V}{\partial x}\right)_{n+\frac{1}{3}}^{m}=\frac{V_{n+1}^{m}-V_{n}^{m}}{\Delta x}
$$

$$
\left(\frac{\partial M}{\partial x}\right)_{n+1}^{m}=\frac{M_{n+1}^{m}-M_{n}^{m}}{\Delta x}
$$

$$
\left(\frac{\partial y}{\partial x}\right)_{n}^{m+1}=\frac{y_{n+1}^{m+1}-y_{n-\frac{1}{2}}^{m+1}}{\Delta x}
$$

$$
\left(\frac{\partial \gamma}{\partial x}\right)_{n}^{m+1}=\frac{\gamma_{n+\frac{1}{2}}^{m+1}-\gamma_{n-1}^{m+1}}{\Delta x} \text {. }
$$

The resulting system of finite difference equations is given below:

$$
\begin{gathered}
y_{n+1}^{m+1}=\left(2 y_{n+1}^{m}-y_{n+\frac{1}{2}}^{m-1}\right)-\left(\frac{c_{n+\frac{1}{3}}}{\mu_{n+1}}\right)\left(y_{n+\frac{1}{3}}^{m}-y_{n+\frac{1}{2}}^{m-1}\right) \Delta t \\
-\frac{\left(V_{n+1}^{m}-V_{n}^{m}\right)}{\mu_{n+1}} \frac{(\Delta t)^{2}}{\Delta x}+P_{n+1}^{m} \frac{(\Delta t)^{2}}{\mu_{n+1}} \\
\gamma_{n+\frac{1}{2}}^{m+1}=\left(2 \gamma_{n+\frac{1}{2}}^{m}-\gamma_{n+\frac{1}{2}}^{m-1}\right)+\frac{M_{n+1}^{m}-M_{n}^{m}}{\left(I_{\mu z}\right)_{n+1}} \frac{(\Delta t)^{2}}{\Delta x}-\frac{V_{n+\frac{1}{3}}^{m}(\Delta t)^{2}}{\left(I_{\mu z}\right)_{n+1}} \\
M_{n}^{m+1}=(E I)_{n} \frac{\gamma_{n+\frac{1}{3}}^{m+1}-\gamma_{n-1}^{m+1}}{\Delta x} \\
V_{n}^{m+1}=(K A G)_{n} \gamma_{n}^{m+1}-(K A G)_{n} \frac{y_{n+\frac{1}{3}}^{m+1}-y_{n-1}^{m+1}}{\Delta x}
\end{gathered}
$$

where

$$
\gamma_{n}^{m}=\frac{1}{2}\left(\gamma_{n+\frac{1}{2}}^{m}+\gamma_{n-\frac{1}{2}}^{m}\right), \quad V_{n+\frac{1}{2}}^{m}=\frac{1}{2}\left(V_{n}^{m}+V_{n+1}^{m}\right)
$$

In order to define completely the motion of the ship hull (non-uniform beam) it is required that initial and boundary conditions be fixed. If we begin our computation when the vessel is at rest (just prior to subjecting it to any force) we have the 
condition at $t_{0}=0, y=\gamma=d y / d t=d \gamma / d t=V=M=0$. At all time, $t$, we have the condition $M_{0}=V_{0}=M_{20}=V_{20}=0$, on the basis of our assumption that the hull is vibrating freely.

The above system of equations (equations (12) to (15)) taken together with the supplementary initial and boundary conditions may be summarized in the following form used in programming the solution on a high-speed digital calculator (UNIVAC System):

$$
\begin{aligned}
& y_{n+1}^{m+1}=\left(1+K_{1}\right)\left(y_{n+\frac{1}{3}}^{m}-y_{n+\frac{1}{3}}^{m-1}\right)+y_{n+\frac{1}{3}}^{m}+K_{2}\left(V_{n+1}^{m}-V_{n}^{m}\right)+K_{3} \\
& \gamma_{n+1}^{m+1}=\left(\gamma_{n+1}^{m}-\gamma_{n+1}^{m-1}\right)+\gamma_{n+\frac{1}{2}}^{m}+K_{5}\left(M_{n+1}^{m}-M_{n}^{m}\right)+K_{6}\left(V_{n+1}^{m}+V_{n}^{m}\right) \\
& M_{n}^{m+1}=K_{4}\left(\gamma_{n+1}^{m+1}-\gamma_{n-1}^{m+1}\right) \\
& V_{n}^{m+1}=K_{7}\left(\gamma_{n+1}^{m+1}+\gamma_{n-\frac{1}{1}}^{m+1}\right)+K_{8}\left(y_{n+\frac{1}{3}}^{m+1}-y_{n-\frac{1}{3}}^{m+1}\right)
\end{aligned}
$$

where $K_{1}, K_{2}, \cdots K_{8}$ are multiplying factors which are, with the exception of $K_{3}$, functions only of the characteristics of the vessel hull and the selected time and space intervals, and which may be precomputed prior to the main calculation. $K_{3}$ may also be precomputed in the case of the application of a constant force; in the case the acting force is a function of time a new set of values $K_{3}$ must be computed for each time interval. Specifically,

$$
\left\{\begin{array}{lll}
K_{1}=-\frac{c_{n+1}}{\mu_{n+1}} \Delta t, & K_{2}=-\frac{1}{\mu_{n+1}} \frac{(\Delta t)^{2}}{\Delta x}, & K_{3}=\frac{P_{n+1}^{m}}{\mu_{n+1}}\left(\Delta t^{2}\right), \\
K_{4}=\frac{(E I)_{n}}{\Delta x}, & K_{6}=\frac{1}{\left(I_{\mu z}\right)_{n+1}} \frac{(\Delta t)^{2}}{\Delta x}, & K_{6}=-\frac{(\Delta t)^{2}}{2\left(I_{\mu z}\right)_{n+1}} \\
K_{7}=\frac{(K A G)_{n}}{2}, & K_{8}=-\frac{(K A G)_{n}}{\Delta x} . &
\end{array}\right.
$$

The initial and boundary conditions are given by the relations,

$$
\left\{\begin{array}{l}
y_{n+1}^{0}=y_{n+\frac{1}{2}}^{-1}=\gamma_{n+\frac{1}{2}}^{0}=\gamma_{n+1}^{-1}=V_{n}^{0}=M_{n}^{0}=0, \\
M_{0}{ }^{m}=M_{20}^{m}=V_{0}^{m}=V_{20}^{m}=0 .
\end{array}\right.
$$

From the above it is also possible to derive the following useful relations for the end-point values of $y$ :

$$
\gamma_{0}{ }^{m}=\gamma_{t}{ }^{m}, \quad \gamma_{20}^{m}=\gamma_{10.5}^{m} ; \quad y_{0}{ }^{m}=y_{3}{ }^{m}-\frac{\Delta x}{2} \gamma_{i}^{m} ; \quad y_{20}^{m}=y_{19.5}^{m}+\frac{\Delta x}{2} \gamma_{19.5}^{m} .
$$

4. Numerical Stability. In order to carry out successfully the solution of a system of partial differential equations such as equations (1) to (4) by finite difference methods, the finite difference equivalent system (12) to (15) must be stable in the sense discussed in [6], [7] and [8]. We will now derive the conditions under which this system of equations will satisfy these stability requirements. The conditions of stability are satisfied if the amplitude of a small disturbance, introduced at any time, $t$, does not increase exponentially with successive time steps. This condition may be stated as follows: If $\delta F(x, t)$ and $\delta F(x, t+\Delta t)$ are values of a variation (or perturbation) of any of the dependent variables $y, \gamma, M$ and $V$ in the system, then it is said to be stable provided $|\delta F(x, t+\Delta t) / \delta F(x, t)| \leqq \mid$. To determine the condi- 
tions for stability we will introduce perturbations $\delta y, \delta \gamma, \delta M$ and $\delta V$ in the dependent variables $y, \gamma, M$ and $V$, respectively. Substituting in equations (12) to (15) we obtain the variational equation system:

$$
\begin{gathered}
\mu_{n+1}\left(\delta y_{n+1}^{m+1}-2 \delta y_{n+1}^{m}+\delta y_{n+1}^{m-1}\right)+c_{n+1}\left(\delta y_{n+1}^{m}-\delta y_{n+1}^{m-1}\right) \Delta t \\
+\frac{(\Delta t)^{2}}{\Delta x}\left(\delta V_{n+1}^{m}-\delta V_{n}^{m}\right)=0 \\
\frac{\left(I_{\mu z}\right)_{n+1}}{(\Delta t)^{2}}\left(\delta \gamma_{n+1}^{m+1}-2 \delta \gamma_{n+\frac{1}{3}}^{m}+\delta \gamma_{n+1}^{m-1}\right)-\frac{\delta M_{n+1}^{m}-\delta M_{n}^{m}}{\Delta x}+\delta V_{n+1}^{m}=0 \\
\frac{(E I)_{n}}{\Delta x}\left(\delta \gamma_{n+1}^{m+1}-\delta \gamma_{n-1}^{m+1}\right)-\delta M_{n}^{m+1}=0 \\
\frac{\delta y_{n+1}^{m+1}-\delta y_{n-1}^{m+1}}{\Delta x}-\delta \gamma_{n}^{m+1}+\frac{\delta V_{n}^{m+1}}{(K A G)_{n}}=0 .
\end{gathered}
$$

We will assume in this analysis that within a small region in the $(\dot{x}, t)$ plane the coefficients $\left(\mu, I_{\mu z}\right.$, etc.) of the variational functions can be treated as constants. A solution of the system of equations (19) to (22) can then be obtained in the form

$$
\left\{\begin{array}{l}
\delta y_{n}{ }^{m}=a e^{i \beta n+\alpha m \Delta t} \\
\delta \gamma_{n}{ }^{m}=b e^{i \beta n+\alpha m \Delta t} \\
\delta M_{n}{ }^{m}=c e^{i \beta n+\alpha m \Delta t} \\
\delta V_{n}{ }^{m}=d e^{i \beta n+\alpha m \Delta t}
\end{array}\right.
$$

where $a, b, c, d$ are real constants and $\alpha$ complex. Substituting (23) in (19) to (22) we obtain a system of linear homogeneous equations for the quantities $a, b, c$ and $d$ which has a non-trivial solution provided the determinant $D$ of the coefficients is identically zero, where

$$
\mid \begin{array}{cccc}
\mu\left(\lambda-2+\lambda^{-1}\right)+c \Delta t\left(1-\lambda^{-1}\right) & 0 & 0 & \frac{(\Delta t)^{2}}{\Delta x}\left(e^{\frac{i \beta}{2}}-e^{-\frac{i \beta}{2}}\right) \\
0 & \frac{I_{\mu z}}{(\Delta t)^{2}}\left(\lambda-2+\lambda^{-1}\right) & -\frac{\left(e^{\frac{i \beta}{2}}-e^{-\frac{i \beta}{2}}\right)}{\Delta x} & 1 \\
0 & \frac{E I}{\Delta x}\left(e^{\frac{i \beta}{2}}-e^{\left.-\frac{i \beta}{2}\right)}\right. & -1 & 0 \\
\frac{\left(e^{\frac{i \beta}{2}}-e^{-\frac{i \beta}{2}}\right)}{\Delta x} & -1 & 0 & \frac{1}{(K A G)}
\end{array}
$$

and where $\lambda=e^{\alpha \Delta t}$. From the above we obtain

$$
\begin{gathered}
{\left[\mu\left(\lambda-2+\lambda^{-1}\right)+c \Delta t\left(1-\lambda^{-1}\right)\right]\left[-1+\frac{E I}{(K A G)(\Delta x)^{2}}\left(2 i \sin \frac{\beta}{2}\right)^{2}\right.} \\
\left.-\frac{I_{\mu z}\left(\lambda-2+\lambda^{-1}\right)}{\left(\overline{K A G}(\Delta t)^{2}\right.}\right] \\
-\frac{(\Delta t)^{2}}{(\Delta x)^{2}}\left(2 i \sin \frac{\beta}{2}\right)^{2}\left[\frac{E I}{(\Delta x)^{2}}\left(2 i \sin \frac{\beta}{2}\right)^{2}-\frac{I_{\mu z}\left(\lambda-2+\lambda^{-1}\right)}{(\Delta t)^{2}}\right]=0
\end{gathered}
$$


If we further assume $\Delta t \ll(\mu / c)$ we obtain,

$$
\left(\frac{\mu \xi}{K A G}+4 r \sin ^{2} \frac{\beta}{2}\right)\left(4 r(E I) \sin ^{2} \frac{\beta}{2}+I_{\mu z} \xi\right)+\mu \xi(\Delta t)^{2}=0,
$$

or

$$
\frac{\mu I_{\mu z}}{K A G} \xi^{2}+\left[4 r \sin ^{2} \frac{\beta}{2}\left(I_{\mu z}+\frac{\mu(E I)}{K A G}\right)+\mu(\Delta t)^{2}\right] \xi+16 r^{2}(E I) \sin ^{4} \frac{\beta}{2}=0
$$

where

$$
\xi=\lambda-2+\lambda^{-1}, \quad r=\frac{(\Delta t)^{2}}{(\Delta x)^{2}} .
$$

Equation (26) above is a fourth degree polynomial equation in $\lambda$. If we designate one of its roots $\lambda_{1}$ we draw the conclusion from the relation $\xi=\lambda-2+\lambda^{-1}$ that $\lambda_{2}=1 / \lambda_{1}$ will be another root. For stability both $\left|\lambda_{1}\right| \leqq 1$ and $\left|\lambda_{2}\right|=$ $\left|1 / \lambda_{1}\right| \leqq 1$. It follows that $\left|\lambda_{1}\right|=\left|\lambda_{2}\right|=1$. Likewise, $\left|\lambda_{3}\right|=\left|\lambda_{4}\right|=1$.

Let $\lambda_{1}=\cos \gamma+i \sin \gamma ; \lambda_{2}=\cos \gamma-i \sin \gamma=1 / \lambda_{1}$, then,

$$
\xi=2(\cos \gamma-1) \text {, }
$$

or

$$
-4 \leqq \xi \leqq 0 .
$$

On the other hand, from equation (26) we obtain

$$
\xi=-\frac{K A G}{2 \mu I_{\mu z}}\left[\left(U S+\mu(\Delta t)^{2}\right)+\left(U^{2} T^{2}+2 \mu(\Delta t)^{2} U S+\mu^{2}(\Delta t)^{4}\right)^{\frac{1}{1}}\right]
$$

and

$$
\xi=-\frac{K A G}{2 \mu I_{\mu z}}\left[\left(U S+\mu(\Delta t)^{2}\right)-\left(U^{2} T^{2}+2 \mu(\Delta t)^{2} U S+\mu^{2}(\Delta t)^{4}\right)^{\frac{1}{3}}\right]
$$

where,

$$
S=\left(I_{\mu z}+\frac{\mu(E I)}{K A G}\right), \quad T=\left(I_{\mu z}-\frac{\mu(E I)}{K A G}\right), \quad U=4 r \sin ^{2} \frac{\beta}{2}
$$

It follows that, for stability, the inequalities

$$
-4 \leqq-\frac{K A G}{2 \mu I_{\mu z}}\left[\left(U S+\mu(\Delta t)^{2}\right)+\left(U^{2} T^{2}+2 \mu(\Delta t)^{2} U S+\mu^{2}(\Delta t)^{4}\right)^{\frac{1}{3}}\right] \leqq 0
$$

and

$$
\text { (31) }-4 \leqq-\frac{K A G}{2 \mu I_{\mu z}}\left[\left(U S+\mu(\Delta t)^{2}\right)-\left(U^{2} T^{2}+2 \mu(\Delta t)^{2} U S+\mu^{2}(\Delta t)^{4}\right)^{\frac{1}{2}}\right] \leqq 0
$$

must hold. It may be seen by examining the expression for $\xi$ that its value is always less than or at most equal to zero and that the left hand inequalities (30) and (31) are satisfied if

$$
\left(U S+\mu(\Delta t)^{2}\right) \frac{K A G}{2 \mu I_{\mu z}} \leqq 2 .
$$


In addition, from the previous discussion it follows that $\Delta t$ must be chosen so that

$$
\Delta t \ll \frac{\mu}{c} .
$$

The above result may be stated as follows:

\section{THEOREM}

The finite difference system, consisting of equations (12) to (15), governing the transient motion of a ship hull, is numerically stable provided the time increment $\Delta t$ is chosen sufficiently small so that $\Delta t \ll \mu / c$ and

$$
(\Delta t)^{2} \leqq \frac{\mu I_{\mu z}(\Delta x)^{2}}{I_{\mu z}(K A G)+\mu(E I)+.25 \mu(\Delta x)^{2}(K A G)}
$$

throughout the range of solution.

Numerical instability is usually accompanied by violent variations in the computed functions which invalidate the solution. This effect is shown in Figure 2. Whereas a time interval satisfying inequalities (32) and (33) guarantees stability, it should be pointed out that the interval need not necessarily be as small as indicated by these inequalities.

5. Computational Procedure. We are now prepared to use the system of finite difference equations $\left(12^{\prime}\right)$ to $\left(15^{\prime}\right)$ and $(16)$ to (18) to calculate the motion of a ship hull in response to a force acting upon it. The physical characteristics of the ship hull and the magnitude of the forces acting upon it (i.e. $\mu, C, P, I_{\mu z},(E I)$ and $(K A G))$ are calculated on the basis of the theory of elasticity from experimentally determined physical quantities. (For instance, see Table 7, [5] for values

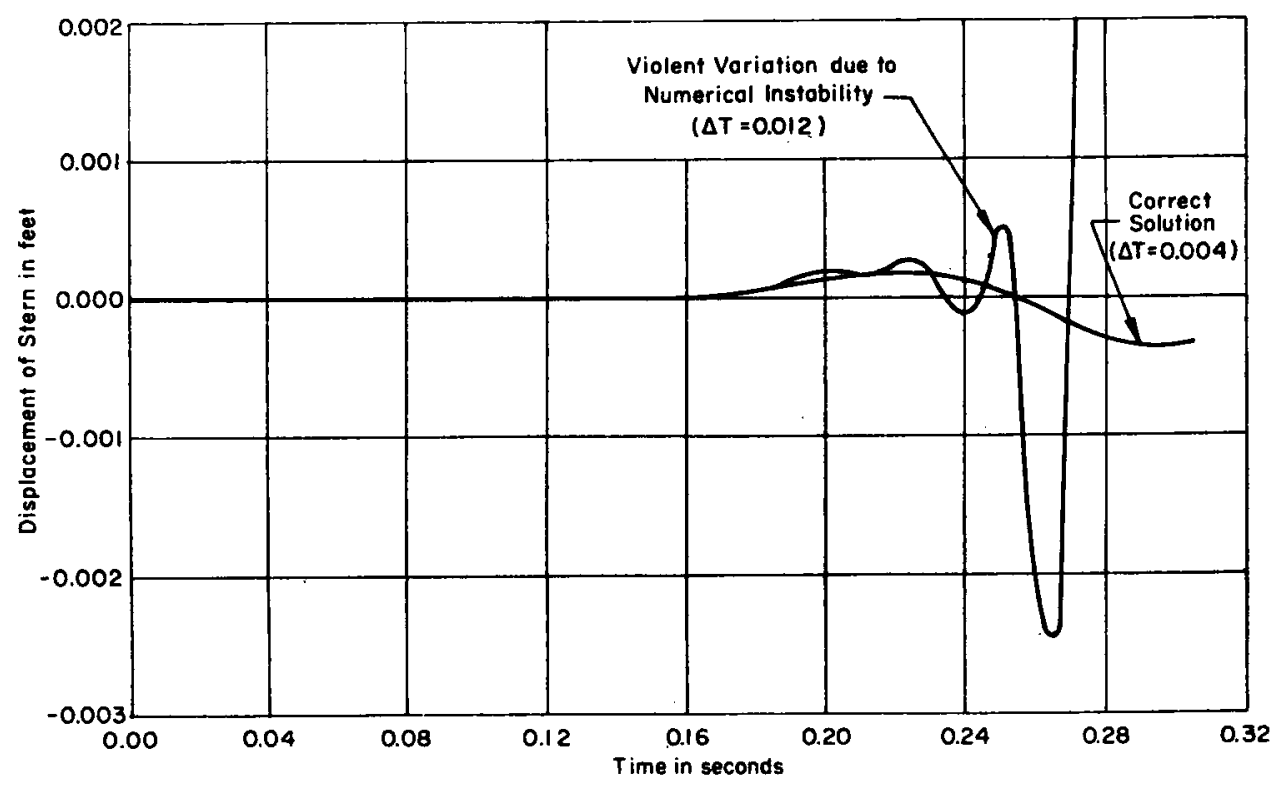

Frg. 2.-Numerical instability resulting from incorrect choice of integration interval. 
of these parameters in the case of the SS Gopher Mariner.) From these the maximum value that can be assigned to $\Delta t$, the time increment, in order to insure numerical stability may be calculated on the basis of the inequalities (32') and (33). Then the coefficients $K_{1}, K_{2}, \cdots K_{8}$ are calculated by use of equations (16). These should be listed at the full-interval positions or at the half-interval positions (See Fig. 1b) as follows:

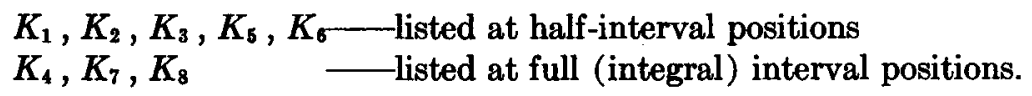

From the known conditions $(y, \gamma, M$, and $V)$ at time $t=0$ (equation 17) we proceed to calculate the values of $y$ and $\gamma$ at $t=\Delta t$, by use of equations $\left(12^{\prime}\right)$ and $\left(13^{\prime}\right)$. We then obtain the values of $M$ and $V$ at $t=\Delta t$ from equations $\left(14^{\prime}\right)$ and $\left(15^{\prime}\right)$, also using the boundary relations $M_{0}=M_{20}=V_{0}=V_{20}=0$. We may now repeat this cycle any number of times, obtaining the values of the variables $y, \gamma, M$ and $V$ at $t=2 \Delta t, 3 \Delta t, \cdots$, etc.-until we reach any desired value of time, $t$.

The above computation procedure was programmed for solution on the UNIVAC system, and trial solutions carried out. The results appear successful in every respect. Calculations at varying time intervals demonstrate the feasibility of producing accurate solutions much beyond engineering requirements.

Applied Mathematics Laboratory

David Taylor Model Basin,

Washington, District of Columbia

1. R. T. MCGoLprick, Calculation of the Response of a Ship Hull to a Transient Load by a Digital Process, David Taylor Model Basin Report 1119, 1957.

2. H. Pouncher, Calculation of Transient Excitation of Ship Hulls by Finite Difference Methods, David Taylor Model Basin Report 1120, 1957.

3. S. TImosenenko, Vibration Problems in Engineering, Van Nostrand, New York, 1928 and 1937.

4. R. T. McGoldrick \& V. L. Russo, “Hull Vibration Investigation on SS Gopher Mariner, Trans. SMAME", v. 64, David Taylor Model Basin Report 1060, 1956.

5. R. T. McGouprick, Calculations for Hull Vibration of the SS Gopher Mariner and Comparison with Experimental Results, David Taylor Model Basin Report 1022, 1956.

6. G. G. O'Brien, M. A. HrMan \& S. Kaplan, "A study of the numerical solution of partial differential equations, “J. Math. Physics, v. 29, 1951, p. 223-251.

7. P. D. Lax \& R. D. RichmyYre, "Stability of difference equations," Communications on Pure and A pplied Mathematics, v. IX, 1956, p. 267-293.

8. G. LUDFord, H. Polacher \& R. J. SHEgrk, "On unsteady flow of compressible viscous fluids," Journal of Applied Physics, v. 24, 1953, p. 490-495. 